\title{
ON A GENERALIZATION OF THE PROBLEM OF QUASI-ANALYTICITY
}

\author{
BY \\ S. MANDELBROJT AND F. E. ULRICH
}

Introduction. The class of all functions analytic in the closed interval $a \leqq x \leqq b$, may be characterized in the following manner: It is the class of all functions $f(x)$ defined and infinitely differentiable (indicated hereafter as i.d. functions) in the interval such that to each function there corresponds a positive constant $k=k(f)$ with the property [5] (1) that

$$
\left|f^{(n)}(x)\right|<k^{n} n !, \quad n \geqq 1 ; a \leqq x \leqq b .
$$

Gevray $\left({ }^{2}\right)$, in studying the heat equation, introduced functions $\Phi(x)$ such that

$$
\left|\Phi^{(n)}(x)\right|<k^{n}(2 n) !, \quad n \geqq 1,
$$

or more generally

$$
\left|\Phi^{(n)}(x)\right|<k^{n} \Gamma(\alpha n), \quad n \geqq 1,
$$

where $\alpha$ is a constant greater than unity. These functions are in general not analytic. In fact, it is possible to construct a function [5] satisfying this last inequality in a closed bounded interval, and such that the function and all its derivatives are zero at one point of the interval, but which is not identically zero in the interval.

In the work to follow we shall denote by $I$ either a closed bounded interval $[a, b]$, or an infinite interval of one of the three forms: $(-\infty, b],(-\infty, \infty)$, $[a, \infty)$. If $\left\{M_{n}\right\}$ is a sequence of positive constants, we shall denote by $C_{\left\{M_{n}\right\}}$ the class of all functions $f(x)$ i.d. in an interval $I$ and such that to each function there corresponds a positive constant $k$ with the property that in $I$

$$
\left|f^{(n)}(x)\right|<k^{n} M_{n}, \quad n \geqq 1 .
$$

The class of analytic functions and the two classes of Gevray mentioned above, each defined in a closed bounded interval, are respectively classes $C_{\{n \mid\}}, C_{\{(2 n) !\}}$ and $C_{\{\Gamma(\alpha n)\}}$.

The class $C_{\{n \mid\}}$ has the property that each function belonging to it such that the function and its derivatives of all orders are zero at one point of the interval is identically zero. Or what is equivalent, two functions each belong-

Presented to the Society, September 10, 1942; received by the editors August 15, 1941.

(1) Numbers in brackets refer to the references listed in the bibliography given at the end of the paper.

(2) See, for instance, S. Mandelbrojt, Rice Institute Pamphlet, vol. 29, 1942. 
ing to this class, and having the same value and the same derivatives of all orders at one point of the interval are identically equal in the interval. A class $C_{\left\{M_{n}\right\}}$ having this property is called quasi-analytic. Thus the classes of Gevray are not quasi-analytic.

The physical character of Gevray's work indicates the importance of knowing necessary and sufficient conditions on the sequence $\left\{M_{n}\right\}$ in order that the class $C_{\left\{M_{n}\right\}}$ will be quasi-analytic. This problem was proposed by Hadamard $\left({ }^{3}\right)$ in 1912. Denjoy [3] gave a sufficient condition and Carleman [2] generalized Denjoy's condition and gave the complete answer to Hadamard's question. The following is Carleman's theorem in a form given by Ostrowski [8]:

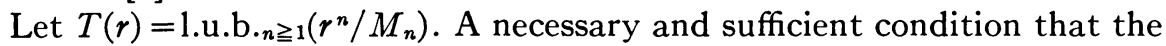
class $C_{\left\{M_{n}\right\}}$ will be quasi-analytic is that $\int_{1}^{\infty} \log T(r) d r / r^{2}=\infty$.

If $I$ is the interval $0 \leqq x \leqq 2 \pi$ and $C_{\left\{M_{n}\right\}}^{*}$ is the class of functions even and periodic with period $2 \pi$ and belonging to $C_{\left\{M_{n}\right\}}$ in $I$, the class $C_{\left\{M_{n}\right\}}$ and the class $C_{\left\{M_{n}\right\}}^{*}$ are quasi-analytic at the same time. In other words, if there exists a function $f(x)$ not identically zero belonging to $C_{\left\{M_{n}\right\}}$ in $I$ such that the function and all its derivatives are zero at a point of $I$, then there exists a function $\Phi(x)$ not identically zero belonging to $C_{\left\{M_{n}\right\}}^{*}$ such that the function and all its derivatives are zero at a point of $I$.

It is known $[4,5]$ that if there exists in $[0,1]$ a function $f(x)$ not identically zero and belonging to $C_{\left\{M_{n}\right\}}$, with $f^{(n)}(0)=0(n \geqq 0)$, then there exists in the same interval a function $\Phi(x)$ not identically zero and belonging to $C_{\left\{M_{n}\right\}}$, with $\Phi^{(n)}(0)=\Phi^{(n)}(1)=0(n \geqq 0)$, and such that $\Phi(x)=\Phi(1-x)$. As a matter of fact, if $C_{\left\{M_{n}\right\}}$ is not quasi-analytic, there exists a function $\Phi(x)$ not identically zero in $[0,1]$ having the prescribed properties and such that $\left|\Phi^{(n)}(x)\right|<M_{n}(n \geqq 1)$, [5]. Thus on writing $\psi(x)=\Phi(x / 2 \pi)$ and continuing this function by periodicity with period $2 \pi$, one sees immediately that if $C_{\left\{M_{n}\right\}}$ is not quasi-analytic, there exists a function $\psi(x)$ belonging to $C_{\left\{M_{n}\right\}}^{*}$ and not identically zero, such that $\psi^{(n)}(0)=\psi^{(n)}(2 \pi)=0(n \geqq 0)$ and such that $\left|\psi^{(n)}(x)\right|<M_{n}(n \geqq 1), x$ in $[0,2 \pi]$; that is, for which the constant $k$ is unity.

It can be shown that if $C_{\left\{M_{n}\right\}}^{*}$ is not quasi-analytic then there exists a function $F(s)=\sum_{m=1}^{\infty} a_{m} / m^{s}, s=\sigma+i t$, where this series converges absolutely in all the plane $\left(\sigma_{A}=\right.$ abscissa of absolute convergence $\left.=-\infty\right)$, the function $F(s)$ having the following properties:

(i) If $M(\sigma)=\sum_{m=1}^{\infty}\left|a_{m}\right| / m^{\sigma}$, then $M(-n) \leqq M, n=1,2, \cdots$.

(ii) $F(-2 n)=0$ for $n>n_{0}>0$.

(iii) $F(s)$ is not identically zero.

Indeed, if $C_{\left\{M_{n}\right\}}^{*}$ is not quasi-analytic, there exists an i.d. function $\Phi(x)=\sum_{m=0}^{\infty} d_{m} \cos m x$, not identically zero, with $\Phi^{(n)}(0)=\Phi^{(n)}(2 \pi)=0(n \geqq 0)$ and such that $\left|\Phi^{(n)}(x)\right|<M_{n}(n \geqq 1)$. We then have

(3) J. Hadamard in a communication to Société Mathématique de France (Comptes Rendus des Séances de la Société Mathématique de France, 1912, p. 28). 


$$
\Phi^{(2 p)}(0)=(-1)^{p} \sum_{m=1}^{\infty} d_{m} m^{2 p}=0,
$$

But

$$
d_{m}=\frac{1}{\pi} \int_{0}^{2 \pi} \Phi(x) \cos m x d x
$$

and so after $q$ integrations by parts we have

so that

$$
d_{m}= \pm \frac{1}{\pi m^{q}} \int_{0}^{2 \pi} \Phi^{(q)}(x) \sin m x_{\sin m x}^{\cos } d x
$$

Therefore $\left({ }^{4}\right)$

$$
\left|d_{m}\right| \leqq \frac{2 M_{q}}{m^{q}}
$$

$$
\left|d_{m}\right| \leqq \frac{2}{\text { l.u.b.q }{ }_{q 1}\left(m^{q} / M_{q}\right)}=\frac{2}{T(m)} .
$$

Now put $a_{m}=d_{m} / 2 c m^{2}(m \geqq 1)$ where $c=\sum_{m=1}^{\infty} 1 / m^{2}$. Then the function $F(s)=\sum_{m=1}^{\infty} a_{m} / m^{s}$ has the desired properties. In the first place

$$
M(-n)=\sum_{m=1}^{\infty}\left|a_{m}\right| m^{n}=\frac{1}{c} \sum_{m=1}^{\infty} \frac{\left|d_{m}\right| m^{n}}{2 m^{2}} \leqq \frac{1}{c} \sum_{m=1}^{\infty} \frac{m^{n-2}}{T(m)} .
$$

But $T(m) \geqq m^{n} / M_{n}$, and so

$$
M(-n) \leqq \frac{M_{n}}{c} \sum_{m=1}^{\infty} 1 / m^{2}=M_{n}
$$

From (1) it follows that, for $n \geqq 2$ :

$$
F(-2 n)=\sum_{m=1}^{\infty} a_{m} m^{2 n}=\frac{1}{2 c} \sum_{m=1}^{\infty} d_{m} m^{2(n-1)}=\frac{(-1)^{n-1}}{2 c} \Phi^{[2(n-1)]}(0)=0 .
$$

Finally, since $\Phi(x)$ is not identically zero, and since $\Phi(0)=0$, the $d_{m}(m \geqq 1)$ are not all zero and, all the $a_{m}$ are not zero, so that $F(s)$ is not identically zero.

The problem of finding conditions on the sequence $\left\{M_{n}\right\}$ in order that the function $F(s)=\sum_{m=1}^{\infty} a_{m} / m^{s}$ will be zero when $F(-2 n)=0\left(n>n_{0}>0\right)$ and $\sum_{m=1}^{\infty}\left|a_{m}\right| m^{n}<M_{n}$, is analogous to, but technically very different from, problems considered by Carlson, Vl. Bernstein [1] and others, concerning entire functions $f(z), z=x+i y$, or functions holomorphic in an angle, and having the following properties (considering only entire functions): If $m(r)$ is a function of $r$ increasing to infinity with $r,|f(z)|<m(r)$ for $|z|=r$ and $f(n)=0(n \geqq 1)$. What then are conditions on the function $m(r)$ in order that $f(z)$ will be identically zero? This problem was solved in many circumstances [1], and the solutions show that the requirement $f(n)=0$ can be replaced by $f\left(\nu_{n}\right)=0$, if the

(4) Such inequalities were first used by Mandelbrojt. See [4 or 5 ]. 
$\left|\nu_{n}\right|$ increase in a suitable manner. In the light of these results it seems natural to suppose that with the same conditions on the sequence $\left\{M_{n}\right\}$ in the problem concerning the series $\sum_{m=1}^{\infty} a_{m} / m^{s}, F(s)$ will be identically zero if we suppose $F\left(-\nu_{n}\right)=0$, the $\nu_{n}$ being real and tending toward infinity, with suitable hypotheses on the mode of increase of the $\nu_{n}$.

It seems reasonable then to expect that if the sequence $\left\{M_{n}\right\}$ satisfies the conditions of quasi-analyticity, or a condition analogous to these, a function belonging to $C_{\left\{M_{n}\right\}}^{*}$ will be zero when the function and its derivatives of orders $\nu_{n}$ are zero at a point, provided the integers $\nu_{n}$ increase in a suitable manner.

It is important to notice that while the conditions on the sequence $\left\{M_{n}\right\}$ are the same in order that a function in either the class $C_{\left\{M_{n}\right\}}$ or the class $C_{\left\{M_{n}\right\}}^{*}$ will be zero when it and its derivatives of all orders are zero at a point, it is impossible to extend to the class $C_{\left\{M_{n}\right\}}$ the notion of quasi-analyticity when only those derivatives of certain orders are zero at a point. The very simple example of the function $f(x) \equiv x$, which belongs to every class and which has derivatives of orders $n \geqq 2$ zero, proves it. This explains why we speak above only of the class $C_{\left\{M_{n}\right\}}^{*}$. Nevertheless, it will also be possible to extend our results to non-periodic functions of a class $C_{\left\{M_{n}\right\}}$ : if the function behaves in a certain manner at infinity.

In a word, we are going to give conditions on the sequence $\left\{M_{n}\right\}$ in order that a function of $C_{\left\{M_{n}\right\}}^{*}$ will be identically zero knowing only that a suitable partial sequence of the derivatives of the function, along with the function itself, is zero at a point. In addition, we shall give conditions on the sequence $\left\{M_{n}\right\}$ in order that a function $f(x)$ of $C_{\left\{M_{n}\right\}}$ in $[0, \infty)$, such that $\int_{0}^{\infty}|f(x)| d x$ $<\infty$, will be identically zero, again knowing only that a suitable partial sequence of its derivatives, along with the function itself, is zero at a point.

If we still suppose that a suitable partial sequence of its derivatives, along with the function itself, is zero at a point, but we no longer suppose that $\int_{0}^{\infty}|f(x)| d x<\infty$, we shall prove that if $\lim \sup _{n \rightarrow \infty}\left|f^{(n)}(0)\right|^{1 / n}<\infty$, then from our conditions on $\left\{M_{n}\right\}$ it follows only that $\lim _{n \rightarrow \infty}\left|f^{(n)}(0)\right|^{1 / n}=0$.

If the sequence of the orders of the derivatives supposed zero at a point is $\left\{\nu_{m}\right\}$, the distribution of the integers $\nu_{m}$ will be characterized by the quantity $G=\lim \sup _{m \rightarrow \infty}\left(\nu_{m} / m\right)$. In all cases our results will be valid if only $G<2$.

\section{PART I}

1. It is well known that the classical problem of the quasi-analyticity of $C_{\left\{M_{n}\right\}}$ is equivalent to the problem of Watson (see $[2,4,8]$ ); that is, the problem of determining necessary and sufficient conditions on the sequence $\left\{M_{n}\right\}$ in order that a function $F(z)$, holomorphic in a half-plane (say $R(z)>1\left({ }^{5}\right)$ ) and satisfying the inequality $|F(z)|<M_{n} /|z|^{n}$, will be identically zero. Our method consists in the very generalization of Watson's problem and the solution of the problem thus generalized.

(s) $R(z)$ denotes the real part of $z$. 
We shall define analytic functions $F_{1}(z)$ and $F_{2}(z)$ as follows: If $f(x)$ belongs to $C_{\left\{M_{n}\right\}}^{*}$,

$$
F_{1}(z)=\int_{0}^{2 \pi} f(x) e^{-x z} d x .
$$

If $f(x)$ belongs to $C_{\left\{M_{n}\right\}}$, where the interval of definition is taken to be $0 \leqq x<\infty$, and $f(x)$ is such that $\int_{0}^{\infty}|f(x)| d x<\infty$, we define

$$
F_{2}(z)=\int_{0}^{\infty} f(x) e^{-x \cdot z} d x
$$

The function $F_{1}(z)$ is an entire function and $F_{2}(z)$ is holomorphic in the right half-plane $R(z)>0$, and bounded there. That is, if $z$ is any point of $R(z)>0,\left|F_{2}(z)\right|$ is less than the constant $\int_{0}^{\infty}|f(x)| d x$. If we integrate $n$ times by parts, we have

$$
\begin{array}{ll}
F_{1}(z)=\left(1-e^{-2 \pi z}\right) \sum_{m=0}^{n-1} \frac{f^{(m)}(0)}{z^{m+1}}+\frac{1}{z^{n}} \int_{0}^{2 \pi} f^{(n)}(x) e^{-x z} d x, & \text { for all } z, \\
F_{2}(z)=\sum_{m=0}^{n-1} \frac{f^{(m)}(0)}{z^{m+1}}+\frac{1}{z^{n}} \int_{0}^{\infty} f^{(n)}(x) e^{-x z} d x, & R(z)>0 .
\end{array}
$$

In the classical case, where all derivatives of $f(x)$ are supposed zero at the origin, the sums appearing in (2) and (3) are zero, which enables one to pass directly to Watson's problem. In that case, for any $\alpha>0$, in the right halfplane $R(z) \geqq \alpha$ both $\left|F_{1}(z)\right|$ and $\left|F_{2}(z)\right|$ are less than $c k^{n} M_{n} /|z|^{n}$, where $c$ is a constant and $k$ depends only on $f(x)$. This is not true, however, in the case when it is only known that a suitable partial sequence of the derivatives of $f(x)$ vanish at the origin.

Along with the hypothesis that the derivatives of $f(x)$ of orders $\nu_{n}$ are zero at the origin, we shall first assume that $\lim \sup _{n \rightarrow \infty}\left|f^{(n)}(0)\right|^{1 / n}<\infty$. This restriction will be removed later.

While, from what was stated in the introduction, it seemed natural to conceive of our problem for the class $C_{\left\{M_{n}\right\}}^{*}$, composed of all even periodic functions belonging to $C_{\left\{M_{n}\right\}}$, and this problem certainly does not have meaning for all functions of this latter class, it will be expedient to begin our study for those functions of $C_{\left\{M_{n}\right\}}$ for which such a study is possible without supposing periodicity. The periodic case will be treated in Part II.

Let us first prove the following simple lemma:

LEMMA I. Let $\left\{\nu_{n}\right\}$ be a sequence of positive integers, and $\left\{\lambda_{n}\right\}$ the sequence of integers complementary to the sequence $\left\{\nu_{n}\right\}$ with respect to the non-negative integers. If

$$
\liminf _{n \rightarrow \infty} \frac{\lambda_{n}}{n} \geqq G^{\prime}>1 \text {, }
$$


then

$$
\limsup _{n \rightarrow \infty} \frac{\nu_{n}}{n} \leqq \frac{G^{\prime}}{G^{\prime}-1} .
$$

Conversely, from (b), with $G^{\prime}>1$, follows (a).

Denote by $M(t)$ the number of integers $\nu_{n} \leqq t$, and by $N(t)$ the number of integers $\lambda_{n} \leqq t$. If $\lambda_{n} \leqq t<\lambda_{n+1}$, then, for $t \geqq \lambda_{1}$

$$
\frac{\lambda_{n}}{n} \leqq \frac{t}{N(t)}<\frac{\lambda_{n+1}}{n}
$$

from which it follows that $\lim _{i n f}(t / N(t))=\liminf _{n \rightarrow \infty}\left(\lambda_{n} / n\right)$. Thus from (a) wehave that $\lim \sup _{t \rightarrow \infty}(N(t) / t) \leqq 1 / G^{\prime}$. On the other hand, $M(t)+N(t)=[t]+1$, where $[t]$ is the greatest integer contained in $t$. But from this it follows that

$$
\liminf _{t \rightarrow \infty} \frac{M(t)}{t}+\limsup _{t \rightarrow \infty} \frac{N(t)}{t}=1 \text {. }
$$

Since $\lim \sup _{t \rightarrow \infty}(N(t) / t) \leqq 1 / G^{\prime}$, we have that $\lim \inf _{t \rightarrow \infty}(M(t) / t) \geqq 1$ $-\left(1 / G^{\prime}\right)=\left(G^{\prime}-1\right) / G^{\prime}$, and (b) follows from this.

The converse also follows immediately from the above considerations.

2. Theorem I. Suppose $f(x)$ belongs to $C_{\left\{M_{n}\right\}}$ in the interval $0 \leqq x<\infty$ and is such that $\int_{0}^{\infty}|f(x)| d x<\infty$. Moreover, suppose lim sup $\sin _{n \rightarrow \infty}\left|f^{(n)}(0)\right|^{1 / n}<\infty$ and $f^{\left(\nu_{m}\right)}(0)=0(m \geqq 1)$. If $\lim \sup _{m \rightarrow \infty}\left(\nu_{m} / m\right)<2$, and if $\int_{1}^{\infty} \log T(r) d r / r^{2}=\infty$, where $T(r)=$ l.u.b. $n \geqq 1\left(r^{n} / M_{n}\right)$, then $f(x)$ is identically zero.

If we denote by $R(<\infty)$ the number $\lim \sup _{n \rightarrow \infty}\left|f^{(n)}(0)\right|^{1 / n}$ and let $P_{\beta}=R+\beta$, where $\beta$ is any positive number, the series $\sum_{m=0}^{\infty} f^{(m)}(0) / z^{m+1}$ converges uniformly in $|z| \geqq P_{\beta}$ to a holomorphic function $\Phi(z)$. Let $\gamma$ be any positive number and denote by $D(\beta, \gamma)$ the region common to $|z| \geqq P_{\beta}$ and $R(z) \geqq \gamma$. Then in $D(\beta, \gamma)$, from (3) we have

$$
F_{2}(z)-\Phi(z)=\frac{1}{z^{n}} \int_{0}^{\infty} f^{(n)}(x) e^{-x z} d x-\sum_{m=n}^{\infty} \frac{f^{(m)}(0)}{z^{m+1}} .
$$

Since $\lim \sup _{n \rightarrow \infty}\left|f^{(n)}(0)\right|^{1 / n}=R$, there is a constant $P>\max (R, 1)$ such that $\left|f^{(n)}(0)\right|<P^{n}$ for all $n \geqq 1$. Now let $\beta$ be chosen so that $P_{\beta}>P>1$. Then in $|z| \geqq P_{\beta}$

$$
\left|\sum_{m=n}^{\infty} \frac{f^{(m)}(0)}{z^{m+1}}\right| \leqq \frac{P^{n}}{|z|^{n}} \sum_{m=0}^{\infty} \frac{P^{m}}{P_{\beta}^{m+1}}=\frac{1}{P_{\beta}-P} \frac{P^{n}}{|z|^{n}}=\frac{\kappa_{1} P^{n}}{|z|^{n}},
$$

where $\kappa_{1}$ is a constant.

On the other hand, $f(x)$ belongs to $C_{\left\{M_{n}\right\}}$ in $0 \leqq x<\infty$ and so $\left|f^{(n)}(x)\right|$ 
$<k^{n} M_{n}(n \geqq 1)$ for $0 \leqq x<\infty$, where $k$ depends only on $f(x)$, from which it follows that

$$
\left|\frac{1}{z^{n}} \int_{0}^{\infty} f^{(n)}(x) e^{-x z} d x\right|<\frac{k^{n} M_{n}}{|z|^{n}} \int_{0}^{\infty}\left|e^{-x z}\right| d x .
$$

Hence for all $z$ in $R(z) \geqq \gamma$,

$$
\left|\frac{1}{z^{n}} \int_{0}^{\infty} f^{(n)}(x) e^{-x z} d x\right|<\frac{k^{n} M_{n}}{|z|^{n}} \int_{0}^{\infty} e^{-\gamma x} d x=\frac{\kappa_{2} k^{n} M_{n}}{|z|^{n}}, \quad n \geqq 1,
$$

where $\kappa_{2}$ is a constant.

From (4), (5) and (6), it results that in $D(\beta, \gamma)$

$$
\left|F_{2}(z)-\Phi(z)\right|<\frac{\kappa_{1} P^{n}+\kappa_{2} k^{n} M_{n}}{|z|^{n}}, \quad n \geqq 1 .
$$

If $\lim \inf _{n \rightarrow \infty}\left(M_{n}\right)^{1 / n}>0$, there is a constant $c$ such that

$$
\left|F_{2}(z)-\Phi(z)\right|<\frac{c^{n} M_{n}}{|z|^{n}}, \quad \quad n \geqq 1,
$$

for all $z$ in $D(\beta, \gamma)$. But since $\int_{1}^{\infty} \log T(r) d r / r^{2}=\infty$, by Carleman's solution of Watson's problem $[4,8], F_{2}(z) \equiv \Phi(z)$ in $D(\beta, \gamma)$.

If $\lim _{\inf _{n \rightarrow \infty}}\left(M_{n}\right)^{1 / n}=0$, to any positive constant $q(<\infty)$ there corresponds a sequence $\left\{n_{i}\right\}$ of integers such that $M_{n_{i}}^{1 / n_{i}}<q(i \geqq 1)$. Therefore in $D(\beta, \gamma)$

$$
\left|F_{2}(z)-\Phi(z)\right|<\frac{\kappa_{1} P^{n_{i}}+\kappa_{2} k^{n_{i}} q^{n_{i}}}{|z|^{n_{i}}}<\frac{Q^{n_{i}}}{|z|^{n_{i}}}, \quad i \geqq 1,
$$

where $Q(<\infty)$ is a constant. If we denote by $\left\{M_{n}^{\prime}\right\}$ the sequence such that $M_{n_{i}}^{\prime}=Q^{n_{i}}(i \geqq 1)$ and $M_{n}^{\prime}=\kappa_{1} P^{n}+\kappa_{2} k^{n} M_{n}, n$ not equal to any $n_{i}$, then in $D(\beta, \gamma)$

$$
\left|F_{2}(z)-\Phi(z)\right|<\frac{M_{n}^{\prime}}{|z|^{n}} .
$$

Now if we let $T_{1}(r)=$ l.u.b. $n \geqq 1\left(r^{n} / M_{n}^{\prime}\right)$, then $T_{1}(r)=\infty$ for $r>Q$. For, if $r>Q$, we have

$$
T_{1}(r) \geqq \underset{i \geqq 1}{\operatorname{lub.b}} \frac{r^{n_{i}}}{M_{n_{i}}^{\prime}}=\underset{i \geqq 1}{1 . u . b .}(r / Q)^{n_{i}}=\infty .
$$

Therefore $\int_{1}^{\infty} \log T_{1}(r) d r / r^{2}=\infty$ and again $F_{2}(z) \equiv \Phi(z)$ in $D(\beta, \gamma)$.

Thus, under the hypotheses of the theorem, we have in all cases that $F_{2}(z) \equiv \Phi(z)$ in $D(\beta, \gamma)$ and hence in the entire region of definition of the functions. 
If we let $\psi(\zeta)=F_{2}(1 / \zeta), \psi(\zeta)$ is holomorphic in $R(\zeta)>0$, and in $|\zeta|<1 / R$,

$$
\psi(\zeta)=\sum_{m=0}^{\infty} f^{(m)}(0) \zeta^{m+1}=\zeta \sum_{m=1}^{\infty} f^{\left(\lambda_{m}\right)}(0) \zeta^{\lambda_{m}}=\zeta \sum_{m=1}^{\infty} a_{m} \zeta^{\lambda_{m}},
$$

where $\left\{\lambda_{m}\right\}$ is the sequence of integers complementary to the sequence $\left\{\nu_{m}\right\}$ with respect to the non-negative integers. By hypotheses $\int_{0}^{\infty}|f(x)| d x<\infty$ and so $\left|F_{2}(z)\right| \leqq \int_{0}^{\infty}|f(x)| d x$ for all $z$ in $R(z)>0$. Therefore we have that $\psi(\zeta)$ is holomorphic and bounded in $R(\zeta)>0$ and is given by (7) in $|\zeta|<1 / R$.

The conclusion of the theorem is now obtained from the following fact, which for later reference will be stated as

LEMMA II. Let the function $F(z)$ be defined by the series $\sum_{m=1}^{\infty} a_{m} z^{\lambda_{m}}$ in $|z|<\rho$ and let $G^{\prime}=\lim \inf _{m \rightarrow \infty}\left(\lambda_{m} / m\right)$. If $F(z)$ is not identically a constant, in each closed angle with vertex at the origin and with opening $2 \pi / G^{\prime}$, one of the two following possibilities must exist. Either

(i) $F(z)$ has a singular point, or

(ii) $F(z)$ is not bounded.

This result is a part of a theorem of Mandelbrojt. For the proof see [6, p. $15 \mathrm{ff}$.$] .$

Since $\left\{\nu_{m}\right\}$ and $\left\{\lambda_{m}\right\}$ are complementary sequences with respect to the non-neg ative integers, and since $\lim \sup _{m \rightarrow \infty}\left(\nu_{m} / m\right)<2$, it follows from Lemma I that $G^{\prime}>2$. Hence there exist closed angles in the right half-plane with vertex at the origin and with opening $2 \pi / G^{\prime}$. Therefore $\psi(\zeta)$ must be identically a constant since neither (i) nor (ii) is true for $\psi(\zeta)$ in the right halfplane. Moreover, since $\psi(0)=0$, this constant must be zero. Therefore $a_{m}=f^{\left(\lambda_{m}\right)}(0)=0$ for all $m \geqq 1$. Thus all derivatives of $f(x)$, along with $f(x)$ itself, are zero at $x=0$ since $f^{\left(\nu_{m}\right)}(0)=0, m \geqq 1$. Then, since $\int_{1}^{\infty} \log T(r) d r / r^{2}=\infty$, the class $C_{\left\{M_{n}\right\}}$ is quasi-analytic and $f(x)$ is identically zero.

3. If the restriction $\int_{0}^{\infty}|f(x)| d x<\infty$ is removed, the following result can be obtained:

Theorem II. Suppose $f(x)$ belongs to $C_{\left\{M_{n}\right\}}$ in $0 \leqq x<\infty$ and $f^{\left(\nu_{m}\right)}(0)=0$ ' $m \geqq 1$. Moreover, suppose $\lim \sup _{n \rightarrow \infty}\left|f^{(n)}(0)\right| 1 / n<\infty$. If $\lim \sup _{m \rightarrow \infty}\left(\nu_{m} / m\right)<2$, and if $\int_{1}^{\infty} \log T(r) d r / r^{2}=\infty$, then $\lim _{n \rightarrow \infty}\left|f^{(n)}(0)\right|^{1 / n}=0$.

In order to prove this theorem we shall use the following lemmas.

Lemma III. Let $\theta(s)$ be defined by the Dirichlet series $\sum_{m=1}^{\infty} d_{m} e^{-\mu_{m} s}$, where $\lim \inf _{m \rightarrow \infty} \mu_{m} / m \geqq L>0$. Let $\epsilon$ be a positive quantity and $s_{1}=\sigma_{1}+i t_{1}$. If $\theta(s)$ is holomorphic in the circle $\left|s-s_{1}\right| \leqq(\pi / L)(1+\epsilon)\left({ }^{6}\right)$ and if $|\theta(s)|<M$ in this circle

${ }^{(6)}$ A function represented by a Dirichlet series, which, of course, we suppose always to have an axis of convergence, will be said to be holomorphic at a point $\sigma_{0}+i t_{0}$, if it is possible to continue analytically the function given by the sum of the series along the line $t=t_{0}$ through the point $\sigma_{0}+i t_{0}$. The value $\theta\left(\sigma_{0}+i t_{0}\right)$ is the value given by this continuation. 
then for each $j=1,2, \cdots$,

$$
\left|d_{j}\right|\left|g_{i}\left(\mu_{j}\right)\right| e^{-\mu_{j} \sigma_{1}}<K_{\epsilon} M,
$$

where $K_{\mathrm{c}}$ is independent of $j, \sigma_{1}$ and $M$, and

$$
g_{j}(z)=z \prod_{n=1, n \neq j}^{\infty}\left(1-z^{2} / \mu_{n}^{2}\right)(7) .
$$

If $\theta(s)$ is bounded in the whole strip $|t|<\pi / 2$, and if $L>2$, since $\mu_{1}>0$ it is then evident that $d_{j}=0$ for every $j=1,2, \ldots$.

This lemma was proved by Mandelbrojt [6, p. $14 \mathrm{ff}$.] and was used by him in the proof of the theorem which is given in the present paper in a modified form as Lemma II. Lemma III was also proved for entire functions by Mandelbrojt and Gergen [7].

From Lemma III results the following lemma which was given by Mandelbrojt in his lectures at Rice Institute and for which we shall now give the proof:

LEMma IV. Let $F(z)$ be given in the neighborhood of the origin by the Taylor series $\sum_{m=1}^{\infty} a_{m} z^{\mu_{m}}$, with $\lim \inf _{m \rightarrow \infty}\left(\mu_{m} / m\right)=D>L$. If $F(z)$ is holomorphic in the sector $|z|<\rho e^{\pi / L},|\arg z|<\pi / L$, then $\lim \sup _{m \rightarrow \infty}\left|a_{m}\right|^{1 / \mu_{m}} \leqq 2^{3} / \rho(8)$.

If we put $z=e^{-s}$, the function $\Phi(s)=F\left(e^{-s}\right)$ is holomorphic for values of $s=\sigma+i t$ such that $\sigma>-\log \rho-\pi / L,|t|<\pi / L$, and in a half-plane $\sigma>c, \Phi(s)$ is given by the series $\sum_{m=1}^{\infty} a_{m} e^{-\mu_{m s}}$.

There exists therefore a constant $\epsilon>0$ such that $\Phi(s)$ is holomorphic in the circle $\left|s-s_{0}\right| \leqq(\pi / D)(1+\epsilon)$, where $s_{0}=-\log \rho$. Hence by Lemma III we have

$$
\left|a_{i}\right|\left|g_{j}\left(\mu_{j}\right)\right|<K_{\epsilon} M \rho^{-\mu_{j}},
$$

where $g_{j}(z)$ is again the function defined in (8) and $M=\max |\Phi(z)|$ in

$$
\left|s-s_{0}\right| \leqq(\pi / D)(1+\epsilon) \text {. }
$$

We now evaluate $g_{j}\left(\mu_{j}\right)$. Since the $\mu_{m}$ are increasing positive integers.

and

$$
\mu_{j+k} \geqq \mu_{j}+k, \quad k>0,
$$

$$
\mu_{j-k} \leqq \mu_{j}-k,
$$

$0 \leqq k<j$

Therefore

(7) We suppose $\mu_{1}>0$, and as usual, to $\mu_{n}$ increasing to infinity.

(8) We again suppose $\mu_{1}>0$. 


$$
\begin{aligned}
\left|g_{j}\left(\mu_{j}\right)\right| & =\mu_{j}\left|\prod_{n=1, n \neq j}^{\infty}\left(1-\mu_{j}^{2} / \mu_{n}^{2}\right)\right| \geqq \mu_{j} \prod_{p=-j+1, p \neq 0}^{\infty}\left|1-\frac{\mu_{i}^{2}}{\left(\mu_{j}+p\right)^{2}}\right| \\
& =\mu_{j} \prod_{m=\mu_{j}-j+1, m \neq \mu_{j}}^{\prime}\left|1-\mu_{j}^{2} / m^{2}\right| \\
& =\mu_{j}\left[\prod_{m=1, m \neq \mu_{j}}^{\infty}\left|1-\mu_{j}^{2} / m^{2}\right|\right]\left[\prod_{m=1}^{\mu_{j}-j}\left|1-\mu_{j}^{2} / m^{2}\right|\right]^{-1}{ }^{(9)} .
\end{aligned}
$$

But since

$$
\begin{aligned}
\mu_{j} \prod_{m=1, m \neq \mu_{j}}^{\infty}\left(1-\mu_{j}^{2} / m^{2}\right) & =\lim _{z \rightarrow \mu_{j}} z \prod_{m=1}^{\infty}\left(1-z^{2} / m^{2}\right) \cdot \frac{\mu_{j}^{2}}{\mu_{j}^{2}-z^{2}} \\
& =\lim _{z \rightarrow \mu_{j}} \frac{\sin \pi z}{\pi} \cdot \frac{\mu_{j}^{2}}{\mu_{j}^{2}-z^{2}},
\end{aligned}
$$

we see that the left member of this last equality is equal to $-\mu_{j} \cos \pi \mu_{j} / 2$ $=(-1)^{\mu_{j}-1} \mu_{j} / 2$.

On the other hand,

$$
\begin{aligned}
\prod_{m=1}^{\mu_{j}-j}\left|1-\mu_{j}^{2} / m^{2}\right| & =\left[\prod_{m=1}^{\mu_{j}-j} \frac{\mu_{j}-m}{m}\right]\left[\prod_{m=1}^{\mu_{j}-j} \frac{\mu_{j}+m}{m}\right] \\
& =\frac{\left(\mu_{j}-1\right) !}{(j-1) !\left(\mu_{j}-j\right) !} \frac{\left(2 \mu_{j}-j\right) !}{\mu_{j} !\left(\mu_{j}-j\right) !}=\frac{j}{\mu_{j}} \frac{\left(2 \mu_{j}-j\right) !}{\left[\left(\mu_{j}-j\right) !\right]^{2} j !} \\
& =\frac{j}{\mu_{j}} \frac{\left(2 \mu_{j}-j\right) !}{\mu_{j} !\left(\mu_{j}-j\right) !} \cdot \frac{\mu_{j} !}{\left(\mu_{j}-j\right) ! j !}=\frac{j}{\mu_{j}} C_{2 \mu_{j-j}{ }^{\mu_{j}} C_{\mu_{j}}^{j}}^{j} \\
& \leqq \frac{j}{\mu_{j}} 2^{2 \mu_{j-j} 2^{\mu_{j}}=\frac{j}{\mu_{j}} 2^{3 \mu_{j}-j} .}
\end{aligned}
$$

From (10) we then have that

$$
\left|g_{j}\left(\mu_{j}\right)\right| \geqq\left(\mu_{j}^{2} / j\right) 2^{j-3 \mu_{j}-1}
$$

From (9) it then follows that

$$
\left|a_{j}\right| \leqq K_{\epsilon} M\left(j / \mu_{j}^{2}\right) 2^{3 \mu j-j-1} \rho^{-\mu j},
$$

and from this the statement of the lemma results immediately.

We are now ready to pass to the proof of Theorem II. It should be noticed that even under the present hypotheses, the function $F_{2}(z)$ defined by (3) exists for each $z$ in $R(z)>0$ and is holomorphic there. For, appears.

(9) If $\mu_{j}=j$, the quantity in the second brackets must be taken equal to unity wherever it 


$$
f(x)=f(0)+\int_{0}^{x} f^{\prime}(t) d t
$$

and $\left|f^{\prime}(x)\right|<k M_{1}$ in $0 \leqq x<\infty$, so that $|f(x)| \leqq|f(0)|+k M_{1} x, 0 \leqq x<\infty$. Therefore $\int_{0}^{\infty} f(x) e^{-x z} d x$ converges uniformly in $R(z) \geqq \alpha>0$ and defines a holomorphic function there. But under the present hypotheses it can no longer be said that $F_{2}(z)$ is bounded in $R(z)>0$ and so it is not possible to draw the same conclusions as in Theorem I. However, the function $\psi(\zeta)=F_{2}(1 / \zeta)$ is holomorphic in $R(\zeta)>0$ and is given by the series $\zeta \sum_{m=1}^{\infty} f^{\left(\lambda_{m}\right)}(0) \zeta^{\lambda_{m}}$ in $|\zeta|<1 / R$ where $R=\lim \sup _{n \rightarrow \infty}\left|f^{(n)}(0)\right|^{1 / n}$. By Lemma I, $\lim \inf _{m \rightarrow \infty}\left(\lambda_{m} / m\right)>2$ since $\lim \sup _{m \rightarrow \infty}\left(\nu_{m} / m\right)<2$. Hence we can apply Lemma IV with $L=2$ to the function $\psi(\zeta)$. The sequence $\left\{\mu_{m}\right\}$ is replaced by the sequence $\left\{\lambda_{m}+1\right\}$, $a_{m}=f^{\left(\lambda_{m}\right)}(0)$ and $\rho$ can be any positive number. Therefore

$$
\limsup _{m \rightarrow \infty}\left|f^{\left(\lambda_{m}\right)}(0)\right|^{1 / \lambda_{m}} \leqq 2^{3} / \rho,
$$

and since $\rho$ is any positive number

$$
\lim _{n \rightarrow \infty}\left|f^{(n)}(0)\right|^{1 / n}=\lim _{m \rightarrow \infty}\left|f^{\left(\lambda_{m}\right)}(0)\right|^{1 / \lambda_{m}}=0 .
$$

4. We now pass to the case in which it is not assumed that lim $\sup _{n \rightarrow \infty}\left|f^{(n)}(0)\right|^{1 / n}<\infty$. In this case the radius of convergence of the series $\sum_{m=1}^{\infty} f^{\left(\lambda_{m}\right)}(0) \zeta^{\lambda_{m}+1}$ may be zero and then the series no longer represents $\psi(\zeta)$. But we shall see that due to the relation which exists between this series and the function, it is possible to consider the series as somewhat analogous to an asymptotic development in order to obtain some properties of $\psi(\zeta)$.

It is useful first to establish some preliminary lemmas.

LEMMA V. If $\Phi(z)$ is not identically zero and is holomorphic in $|z| \leqq 1$ except possibly at $z=-1$, and is bounded in this circle, then

$$
\int_{-\pi}^{\pi} \log \left|\Phi\left(e^{i \theta}\right)\right| d \theta>-\infty .
$$

This lemma is well known. A proof is given by Ostrowski [8].

Let $\left\{N_{n}\right\}$ be a sequence of positive numbers and $\left\{\mu_{n}\right\}$ a sequence of positive numbers increasing to infinity. If $\omega$ is any positive number, we define

$$
\tau_{\omega}(r)=\underset{n \geqq 1}{\text { l.u.b. }} \frac{r^{\omega \mu_{n}}}{N_{n}} .
$$

LeMMA VI. Let $\left\{N_{n}\right\}$ be a sequence of positive numbers and $\left\{\mu_{n}\right\}$ a sequence of positive numbers increasing to infinity. Suppose $A(\xi)$ is holomorphic and bounded in $R(\xi) \geqq 0$, and 


$$
|A(\xi)|<\frac{k^{\omega \mu_{n}} N_{n}}{|\xi|^{\omega \mu_{n}}}, \quad n \geqq 1, \text { in } R(\xi) \geqq 0,
$$

where $k$ is a constant. If $\int_{1}^{\infty} \log \tau_{\omega}(r) d r / r^{2}=\infty$, then $A(\xi) \equiv 0$.

From (11) we have

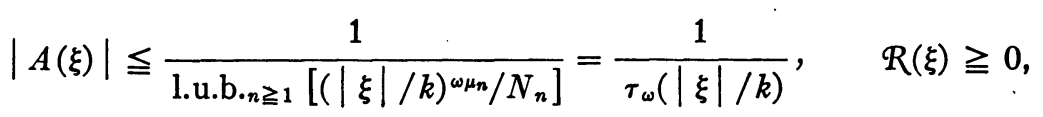

and hence

$$
\log |A(\xi)| \leqq-\log \tau_{\omega}(|\xi| / k) .
$$

Now let $\xi=(1-z) /(1+z)$ and $B(z)=A[(1-z) /(1+z)] . B(z)$ is then holomorphic in $|z| \leqq 1$ except possibly at $z=-1$, and is bounded in this circle. If $A(\xi)$ were not identically zero, $B(z)$ would not be identically zero and by Lemma $\mathrm{V}$ we would have

$$
\int_{-\pi}^{\pi} \log \left|B\left(e^{i \theta}\right)\right| d \theta=\int_{-\pi}^{\pi} \log \left|A\left(\frac{1-e^{i \theta}}{1+e^{i \theta}}\right)\right| d \theta>-\infty .
$$

It would then follow from (12) that

$$
\begin{aligned}
2 \int_{0}^{\pi} \log \tau_{\omega}\left(\frac{\left|1-e^{i \theta}\right|}{k\left|1+e^{i \theta}\right|}\right) d \theta & =\int_{-\pi}^{\pi} \log \tau_{\omega}\left(\frac{\left|1-e^{i \theta}\right|}{k\left|1+e^{i \theta}\right|}\right) d \theta \\
& \leqq-\int_{-\pi}^{\pi} \log \left|A\left(\frac{1-e^{i \theta}}{1+e^{i \theta}}\right)\right| d \theta<\infty
\end{aligned}
$$

But on letting $(1 / k)\left|\left(1-e^{i \theta}\right) /\left(1+e^{i \theta}\right)\right|=(1 / k) \tan (\theta / 2)=r$ we see that the first integral is equal to

$$
2 k \int_{0}^{\infty} \frac{\log \tau_{\omega}(r)}{1+r^{2} k^{2}} d r,
$$

and since $\lim _{r \rightarrow \infty}\left[r^{2} /\left(1+r^{2} k^{2}\right)\right]=1 / k^{2}$, we would have

$$
\int_{1}^{\infty} \frac{\log \tau_{\omega}(r)}{r^{2}} d r<\infty,
$$

which is contrary to hypotheses. Hence it must be true that $A(\xi) \equiv 0$.

We shall now prove the following lemma concerning series which are analogous to an asymptotic series. This lemma is a generalization of the theorem of Mandelbrojt which is given in the present paper as Lemma III.

LEMMA VII. If the sequence $\left\{d_{n}\right\}$ is such that there exist a sequence $\left\{N_{n}\right\}$ of positive numbers, a sequence $\left\{\mu_{n}\right\}$ of increasing positive numbers with $\lim \inf _{n \rightarrow \infty}\left(\mu_{n} / n\right)=G^{\prime}>2, a$ positive quantity $\omega<1-2 / G^{\prime}$, the two sequences and 
the quantity $\omega$ related by $\int_{1}^{\infty} \log \tau_{\omega}(r) d r / r^{2}=\infty$, and a function $\theta(s), s=\sigma+i t$, holomorphic and bounded for $|t|<\pi / 2$, satisfying for every $0<\delta<1$ the inequalities

$$
\left|\theta(s)-\sum_{m=1}^{n} d_{m} e^{-\mu_{m} s}\right|<L_{\delta} N_{n} e^{-\mu_{n} \sigma}, \quad n \geqq 1,|t|<\pi(1-\delta) / 2, \sigma>\sigma_{\delta},
$$

where $L_{\delta}$ and $\sigma_{\delta}$ depend only on $\delta$, then $d_{n}=0(n \geqq 1)$.

The proof has some points in common with the proof of the theorem of Mandelbrojt given as Lemma III. The function $g_{j}(z)$ given by $(8)$ is entire and odd. Hence its expansion in powers of $z$ contains only odd powers:

$$
g_{j}(z)=\sum_{p=0}^{\infty} C_{2 p+1}^{(j)} z^{2 p+1}
$$

In the paper by Mandelbrojt and Gergen [7], it was proved that

$$
\left|C_{2 p+1}^{(j)}\right|<\frac{A_{\epsilon}}{(2 p+1) !}\left[\frac{\pi}{G^{\prime}}(1+\epsilon)^{2 p+1}\right]
$$

where $A_{\epsilon}$ depends only on. $\epsilon$.

Define the function $\theta_{j}(s)$ as follows:

$$
\theta_{j}(s)=\sum_{p=0}^{\infty} C_{2 p+1}^{(j)} \theta^{(2 p+1)}(s) .
$$

Let $\epsilon>0$ and a positive $\delta<1$ be chosen so that $\omega^{\prime}=1-\left(2 / G^{\prime}\right)(1+\epsilon)-\delta>\omega>0$. We shall now prove that the series defining $\theta_{j}(s)$ converges uniformly and absolutely in the strip $S_{\epsilon}:|t|<\pi\left(\omega^{\prime}+\delta\right) / 2$. If $s$ is any point of $S_{\epsilon}$, each point $u$ of the circumference $\gamma:|u-s|=\pi(1+\epsilon) / G^{\prime}$ is in the strip $|t|<\pi / 2$. Then by Cauchy's integral formula,

$$
\left|\theta^{(2 p+1)}(s)\right| \leqq \frac{(2 p+1) !}{2 \pi} \int_{\gamma} \frac{|\theta(u)||d u|}{|u-s|^{2 p+2}} \leqq \frac{(2 p+1) ! M}{\left[\pi(1+\epsilon) / G^{\prime}\right]^{2 p+1}},
$$

where $M$ is the bound on $\theta(s)$ in $|t|<\pi / 2$. Therefore from this inequality and (13) with $\epsilon$ replaced by $\epsilon / 2$, we have that in $S_{\epsilon}$

$$
\left|C_{2 p+1}^{(j)} \theta^{(2 p+1)}(s)\right| \leqq M A_{\epsilon / 2}\left[\frac{1+\epsilon / 2}{1+\epsilon}\right]^{2 p+1},
$$

and so the series defining $\theta_{j}(s)$ converges uniformly and absolutely. Moreover it also follows from this last inequality that

$$
\left|\theta_{j}(s)\right| \leqq M_{\epsilon} A_{\epsilon / 2} \sum_{p=0}^{\infty}\left(\frac{1+\epsilon / 2}{1+\epsilon}\right)^{2 p+1}=K_{\epsilon} M
$$

for each $s$ in $S_{\epsilon}$, where $K_{\epsilon}$ depends only on $\epsilon$. 
If we let

$$
H_{n}(s)=\theta(s)-\sum_{m=1}^{n} d_{m} e^{-\mu_{m} s},
$$

we have by hypothesis that in $|t|<\pi(1-\delta) / 2, \sigma>\sigma_{\delta}$,

$$
\left|H_{n}(s)\right|<L_{\delta} N_{n} e^{-\mu_{n} \sigma}, \quad n \geqq 1 .
$$

Let $S_{\epsilon, \delta}$ be the half-strip defined by $|t|\left\langle\pi \omega^{\prime} / 2, \sigma>\sigma_{\delta}+\pi(1+\epsilon) / G^{\prime}=\sigma_{\delta}^{\prime}\right.$. If $s$ is any point of the half-strip $S_{\epsilon, \delta}$, each point of the circumference $\gamma$ will be a point of the half-strip $|t|\left\langle\pi(1-\delta) / 2, \sigma>\sigma_{\delta}\right.$. Then by the Cauchy integral formula for $H_{n}^{(2 p+1)}(s)$ we have that in $S_{\epsilon, \delta}$

$$
\left|\theta^{(2 p+1)}(s)+\sum_{m=1}^{n} d_{m} \mu_{m}^{2 p+1} e^{-\mu_{m} s}\right|<\frac{(2 p+1) ! L_{\delta} N_{n} e^{-\mu_{n} \sigma}}{\left[\pi(1+\epsilon) / G^{\prime}\right]^{2 p+1}} .
$$

This inequality along with (13) yields that in $S_{\epsilon, \delta}$

$$
\left|C_{2 p+1}^{(j)}\left[\theta^{(2 p+1)}(s)+\sum_{m=1}^{n} d_{m} \mu_{m}^{2 p+1} e^{-\mu_{m} s}\right]\right|<A_{\epsilon / 2} L_{\delta} N_{n} e^{-\mu_{n}^{\sigma}}\left(\frac{1+\epsilon / 2}{1+\epsilon}\right)^{2 p+1} .
$$

Now consider the series

$$
\sum_{p=0}^{\infty} C_{2 p+1}^{(j)}\left[\theta^{(2 p+1)}(s)+\sum_{m=1}^{n} d_{m} \mu_{m}^{2 p+1} e^{-\mu_{m} s}\right] .
$$

By (15) this series converges uniformly and absolutely in $S_{\epsilon, \delta}$ and in this half-strip is equal to

$$
\theta_{j}(s)+\sum_{m=1}^{n} d_{m} e^{-\mu_{m} s}\left(\sum_{p=0}^{\infty} C_{2 p+1}^{(j)} \mu_{m}^{2 p+1}\right)=\theta_{j}(s)+\sum_{m=1}^{n} d_{m} g_{j}\left(\mu_{m}\right) e^{-\mu_{m} s} .
$$

But since $g_{j}\left(\mu_{m}\right)=0$ for $m \neq j$, if $n \geqq j$, this last expression becomes $\theta_{j}(s)$ $+d_{j} g_{j}\left(\mu_{j}\right) e^{-\mu_{j} s}$, and again from (15) it follows that in $S_{\epsilon, \delta}$

$$
\begin{array}{r}
\left|\theta_{j}(s)+d_{j} g_{j}\left(\mu_{j}\right) e^{-\mu_{j} s}\right| \leqq A_{\epsilon / 2} L_{\delta} N_{n} e^{-\mu_{n} \sigma} \sum_{p=0}^{\infty}\left(\frac{1+\epsilon / 2}{1+\epsilon}\right)^{2 p+1}=L_{\delta} K_{\epsilon}^{\prime} N_{n} e^{-\mu_{n} \sigma}, \\
n \geqq j,
\end{array}
$$

where $K_{\epsilon}^{\prime}$ depends only on $\epsilon$.

Let $\eta=\eta_{1}+\eta_{2} i=s / \omega^{\prime}$. Then if we let $E_{j}(\eta)=\theta_{j}\left(\eta \omega^{\prime}\right)$, we have that in $\eta_{1}>\sigma_{\delta}^{\prime} / \omega^{\prime},\left|\eta_{2}\right|<\pi / 2$

$$
\left|E_{j}(\eta)+d_{j} g_{j}\left(\mu_{j}\right) e^{-\mu_{j} \omega^{\prime} \eta}\right|<L_{\delta} K_{\epsilon}^{\prime} N_{n} e^{-\mu_{n} \omega^{\prime} \eta_{1}}, \quad n \geqq j .
$$

Now let $\xi=e^{\eta}$ and denote by $A_{j}(\xi)$ the function corresponding by this transformation to $E_{j}(\eta)+d_{j} g_{j}\left(\mu_{j}\right) e^{-\mu_{j} \omega^{\prime} \eta}$. We then have in the domain defined by $R(\xi)>0,|\xi|>e^{\sigma^{\prime} \delta / \omega^{\prime}}$, since $|\xi|=e^{\eta_{1}}$, 


$$
\left|A_{j}(\xi)\right|<\frac{L_{\delta} K_{\epsilon}^{\prime} N_{n}}{|\xi|^{\prime} \mu_{n}},
$$

But then there exists a constant $k>1$ such that for every $1 \leqq n<j$

$$
\left|A_{j}(\xi)\right|<\frac{L_{\delta} K_{\epsilon}^{\prime} k^{\omega^{\prime} \mu_{n}} N_{n}}{|\xi|^{\omega^{\prime} \mu_{n}}}
$$

in $R(\xi)>0,|\xi|>e^{\sigma^{\prime} \delta / \omega^{\prime}}$. Thus we have that

in $R(\xi)>0,|\xi|>e^{\sigma^{\prime} \delta / \omega^{\prime}}$.

$$
\left|A_{j}(\xi)\right|<\frac{L_{\delta} K_{\epsilon}^{\prime} k^{\omega^{\prime} \mu_{n}} N_{n}}{|\xi|^{\omega^{\prime} \mu_{n}}}, \quad n \geqq 1,
$$

Now let

$$
A(\xi)=A_{j}(a+\xi) / L_{\delta} K_{\epsilon}^{\prime},
$$

where $a=e^{\sigma^{\prime} \delta / \omega^{\prime}}+1 . A(\xi)$ is holomorphic in $R(\xi) \geqq 0$ and from (14) it follows that it is bounded in this closed half-plane. From the above inequality we have that

$$
|A(\xi)|=\frac{\left|A_{j}(a+\xi)\right|}{L_{\delta} K_{\epsilon}^{\prime}} \leqq \frac{k^{\omega^{\prime} \mu_{n}} N_{n}}{|\xi+a|^{\omega^{\prime} \mu_{n}}}<\frac{k^{\omega^{\prime} \mu_{n}} N_{n}}{|\xi|^{\omega^{\prime} \mu_{n}}}, \quad n \geqq 1,
$$

in $R(\xi) \geqq 0$, since $a$ is a real positive quantity.

Since $\omega^{\prime}>\omega$, if $\int_{1}^{\infty} \log \tau_{\omega}(r) d r / r^{2}=\infty$, then $\int_{1}^{\infty} \log \tau_{\omega^{\prime}}(r) d r / r^{2}=\infty$. Thus all the conditions of Lemma VI are satisfied by the function $A(\xi)$ and the sequences $\left\{\mu_{n}\right\}$ and $\left\{N_{n}\right\}$, where the $\omega$ of that lemma becomes the present $\omega^{\prime}$. Therefore $A(\xi) \equiv 0$, and we have that $A_{j}(\xi) \equiv 0$. From this it follows that

$$
\theta_{j}(s) \equiv-d_{j} g_{j}\left(\mu_{j}\right) e^{-\mu_{j} s} .
$$

From (14) we then have that

$$
\left|d_{j}\right|\left|g_{j}\left(\mu_{j}\right)\right| e^{-\mu_{j} \sigma}<M K_{\epsilon}
$$

for all $\sigma$. And since this inequality holds for all $\sigma$, and since $\mu_{1}>0$, it follows that $d_{j}=0$ for $j=1,2, \cdots$. With this the lemma is proved.

Let there be given a sequence $\left\{M_{n}\right\}$ of positive numbers, a sequence $\left\{\nu_{n}\right\}$ of positive integers and a positive constant $\omega$. We shall define

$$
T_{\omega}(r)=\underset{n \geqq 1}{\operatorname{lib} . \text {. }} \frac{r^{\omega \lambda_{n}}}{M_{\lambda_{n}+1}},
$$

where $\left\{\lambda_{n}\right\}$ is the sequence of positive integers complementary to the sequence $\left\{\nu_{n}\right\}$ with respect to the non-negative integers.

Theorem III. Suppose $f(x)$ belongs to $C_{\left\{M_{n}\right\}}$ in $0 \leqq x<\infty$ and is such that $\int_{0}^{\infty}|f(x)| d x<\infty$. Moreover, suppose $f^{\left(\nu_{n}\right)}(0)=0(n \geqq 1)$. If $G=\lim \sup _{n \rightarrow \infty}\left(\nu_{n} / n\right)$ 
$<2$, and if there exists a positive constant $\omega<2 / G-1$ such that $\int_{0}^{\infty} \log T_{\omega}(r) d r / r^{2}$ $=\infty$, then $f(x)$ is identically zero.

We shall again consider $F_{2}(z)$ defined by (3), but now in the half-plane $R(z) \geqq \alpha>0$. We then have in this half-plane

$$
\left|F_{2}(z)-\sum_{m=0}^{n-1} \frac{f^{(m)}(0)}{z^{m+1}}\right| \leqq \frac{1}{|z|^{n}} \int_{0}^{\infty}\left|f^{(n)}(x) e^{-x z}\right| d x .
$$

Since $f(x)$ belongs to $C_{\left\{M_{n}\right\}}$ in $0 \leqq x<\infty$, it follows that

$$
\left|F_{2}(z)-\sum_{m=0}^{n-1} \frac{f^{(m)}(0)}{z^{m+1}}\right|<\frac{c k^{n} M_{n}}{|z|^{n}}, \quad n \geqq 1,
$$

in $R(z) \geqq \alpha$, where $c$ is a constant.

If we let $z=e^{s}, s=\sigma+i t$, and $\Psi(s)=F_{2}\left(e^{s}\right)$, on recalling that $f^{\left(v_{n}\right)}(0)=0$ $(n \geqq 1)$, the last inequality becomes

$$
\left|\Psi(s)-\sum_{m=1}^{n} f^{\left(\lambda_{m}\right)}(0) e^{-\left(\lambda_{m}+1\right) s}\right|<c k^{\lambda_{n}+1} M_{\lambda_{n}+1} e^{-\left(\lambda_{n}+1\right) \sigma}
$$

in $D_{\alpha}$, where $D_{\alpha}$ is the image of $R(z) \geqq \alpha$ by the transformation $z=e^{s}$. Here $\left\{\lambda_{n}\right\}$ is the sequence of integers complementary to the sequence $\left\{\nu_{n}\right\}$ with respect to the non-negative integers. Let $G^{\prime}=\lim \inf _{n \rightarrow \infty}\left(\lambda_{n} / n\right)$. If we let $\mu_{n}=\lambda_{n}+1$, it is evident that $G^{\prime}=\lim \inf _{n \rightarrow \infty}\left(\mu_{n} / n\right)$. Since by hypotheses $G<2$, we have by Lemma I that $G^{\prime}>2$.

To each $0<\delta<1$, there corresponds a number $\sigma_{\delta}$ such that each point of the half-strip $|t|<\pi(1-\delta) / 2, \sigma>\sigma_{\delta}$ is a point of $D_{\alpha}$. Therefore from (16) we have that in this half-strip

$$
\left|\Psi(s)-\sum_{m=1}^{n} f^{\left(\lambda_{m}\right)}(0) e^{-\mu_{m} s}\right|<N_{n} e^{-\mu_{n} \sigma}, \quad n \geqq 1,
$$

where $N_{n}=c k^{\mu_{n}} M_{\mu_{n}}$.

We also have

$$
\begin{aligned}
\tau_{\omega}(r)=\lim _{n \geqq 1} \text {.u.b. } \frac{r^{\omega \mu_{n}}}{N_{n}} & =\text { l.u.b. } \frac{r^{\omega\left(\lambda_{n}+1\right)}}{c k^{\lambda_{n}+1} M_{\lambda_{n}+1}}=\frac{r^{\omega}}{c k} \operatorname{liu}_{n \geqq 1} \text {.b. } \frac{r^{\omega \lambda_{n}}}{M_{\lambda_{n}+1} k^{\lambda_{n}}} \\
& =\frac{r^{\omega}}{c k} T_{\omega}\left(\frac{r}{k^{1 / \omega}}\right) .
\end{aligned}
$$

From this it is then evident that the divergence of $\int_{1}^{\infty} \log \tau_{\omega}(r) d r / r^{2}$ follows from the divergence of $\int_{1}^{\infty} \log T_{\omega}(r) d r / r^{2}$.

Thus $\Psi(s)$ satisfies all the conditions on the function $\theta(s)$ in Lemma VII, where $f^{\left(\lambda_{n}\right)}(0)=d_{n}$. Hence by this lemma $f^{\left(\lambda_{n}\right)}(0)=0, n \geqq 1$. But then all the derivatives of $f(x)$ as well as the function itself are zero at $x=0$. 
On the other hand, since $\omega<2 / G-1=1-2 / G^{\prime}<1$, if $r>1$ :

$$
\begin{aligned}
T(r) & =\underset{n \geqq 1}{\text { l.u.b. }} \frac{r^{n}}{M_{n}} \geqq \underset{n \geqq 1}{\text { l.u.b. }} \frac{r^{\lambda_{n}+1}}{M_{\lambda_{n}+1}} \geqq \underset{n \geqq 1}{\text { l.u.b. }} \frac{r^{\omega\left(\lambda_{n}+1\right)}}{M_{\lambda_{n}+1}}=r^{\omega} \operatorname{liu}_{n \geqq 1} \frac{r^{\omega \lambda_{n}}}{M_{\lambda_{n}+1}} \\
& =r^{\omega} T_{\omega}(r) .
\end{aligned}
$$

Therefore from the divergence of $\int_{1}^{\infty} \log T_{\omega}(r) d r / r^{2}$ results the divergence of $\int_{1}^{\infty} \log T(r) d r / r^{2}$, and so under our present hypotheses the class $C_{\left\{M_{n}\right\}}$ is quasianalytic. Therefore $f(x) \equiv 0$ and the theorem is proved.

\section{PART II}

5. If $f(x)$ belongs to $C_{\left\{M_{n}\right\}}^{*}, G=\lim \sup _{n \rightarrow \infty}\left(\nu_{n} / n\right)$ is always less than or equal to 2. For, the sequence $\left\{\nu_{n}\right\}$ of the orders of the derivatives supposed zero at the origin must contain all the odd integers, since all the odd derivatives are zero there. If $\lim \sup _{n \rightarrow \infty}\left|f^{(n)}(0)\right|^{1 / n}<\infty$ with no further restrictions on $G$, we will prove that $f(x)$ is a trigonometric polynomial

Theorem IV. If $f(x)$ belongs to $C_{\left\{M_{n}\right\}}^{*}$ where $\int_{1}^{\infty} \log T(r) d r / r^{2}=\infty$, and if $\lim \sup _{n \rightarrow \infty}\left|f^{(n)}(0)\right|^{1 / n}<\infty$, then $f(x)$ is a trigonometric polynomial.

In this case we consider the function $F_{1}(z)$ defined by (2)

$$
F_{1}(z)=\int_{0}^{2 \pi} f(x) e^{-x z} d x=\left(1-e^{-2 \pi z}\right) \sum_{m=0}^{n-1} \frac{f^{(m)}(0)}{z^{m+1}}+\frac{1}{z^{n}} \int_{0}^{2 \pi} f^{(n)}(x) e^{-x z} d x .
$$

Suppose

Then

$$
f(x)=\sum_{m=0}^{\infty} a_{m} \cos m x
$$

$$
F_{1}(z)=\sum_{m=0}^{\infty} a_{m} \int_{0}^{2 \pi} e^{-x z} \cos m x d x=z\left(1-e^{-2 \pi z}\right) \sum_{m=0}^{\infty} \frac{a_{m}}{z^{2}+m^{2}} .
$$

The above termwise integration is valid and the series obtained converges uniformly in any bounded closed region; that is, the remainder $\sum_{m=n}^{\infty} a_{m} /\left(z^{2}+m^{2}\right)$ tends uniformly to zero as $n$ tends to infinity. As in the case of Part I, since $\int_{1}^{\infty} \log T(r) d r / r^{2}=\infty, F_{1}(z)$ is given by

$$
F_{1}(z)=\left(1-e^{-2 \pi z}\right) \sum_{m=0}^{\infty} \frac{f^{(m)}(0)}{z^{m+1}}
$$

in $|z|>R$, where $R=\lim \sup _{n \rightarrow \infty}\left|f^{(n)}(0)\right|^{1 / n}<\infty$. But this latter series converges to a holomorphic function in $|z|>R$ and so it follows from (17) that $a_{m}=0$ for $m>R$. This proves the theorem.

6. In the case in which it is no longer assumed that $\lim \sup _{n \rightarrow \infty}\left|f^{(n)}(0)\right|^{1 / n}$ $<\infty$, we have the analogue of Theorem III of Part I : 
Theorem V. Suppose $f(x)$ belongs to $C_{\left\{M_{n}\right\}}^{*}$ and $f^{\left(\nu_{n}\right)}(0)=0(n \geqq 1)$. If $G=\lim \sup _{n \rightarrow \infty}\left(\nu_{n} / n\right)<2$ and if there exists a positive constant $\omega<2 / G-1$ such that $\int_{1}^{\infty} \log T_{\omega}(r) d r / r^{2}=\infty$, then $f(x)$ is identically zero.

Here again we consider the function $F_{1}(z)$ and from (2) we have

$$
\frac{F_{1}(z)}{1-e^{-2 \pi z}}-\sum_{m=0}^{n-1} \frac{f^{(m)}(0)}{z^{m+1}}=\frac{1}{z^{n}\left(1-e^{-2 \pi z}\right)} \int_{0}^{2 \pi} f^{(n)}(x) e^{-x z} d x
$$

Then in $R(z) \geqq \gamma>0$,

$$
\left|\frac{F_{1}(z)}{1-e^{-2 \pi z}}-\sum_{m=0}^{n-1} \frac{f^{(m)}(0)}{z^{m+1}}\right|<\frac{c^{\prime} k^{n} M_{n}}{|z|^{n}}
$$

where $c^{\prime}$ is a constant.

If we let $z=e^{s}$ and $\Psi(s)=F_{1}\left(e^{s}\right) /\left(1-e^{-2 \pi e^{s}}\right)$, on recalling that $f^{\left(\nu_{n}\right)}(0)=0$ $(n \geqq 1)$, this last inequality becomes

$$
\left|\Psi(s)-\sum_{m=1}^{n} f^{\left(\lambda_{m}\right)}(0) e^{-\left(\lambda_{m}+1\right) s}\right|<c^{\prime} k^{\lambda_{n}+1} M_{\lambda_{n}+1} e^{-\left(\lambda_{n}+1\right) \sigma} .
$$

But this is precisely inequality (16) with $c$ replaced by $c^{\prime}$. Therefore in this case also the theorem now follows immediately from Lemma VII.

\section{BibLIOGRAPHY}

1. Vladimir Bernstein, Leçons sur les Progrès Récents de la Théorie des Séries de Dirichlet, Gauthier-Villars, Paris, 1933.

2. T. Carleman, Les Fonctions Quasi-Analytiques, Gauthier-Villars, Paris, 1926.

3. A. Denjoy, Sur les fonctions quasi-analytiques de variable réelle, Comptes Rendus de l'Académie des Sciences, Paris, vol. 173 (1921), p. 1329.

4. S. Mandelbrojt, Séries de Fourier et Classes Quasi-Analytiques de Fonctions, GauthierVillars, Paris, 1935.

5. — Rice Institute Pamphlet, vol. 29, 1942.

6. - Séries Lacunaires, Actualités Scientifiques et Industrielles, Exposés sur la théorie des fonctions, 1936.

7. S. Mandelbrojt and J. J. Gergen, On entire functions defined by a Dirichlet series, American Journal of Mathematics, vol. 53 (1931), p. 1.

8. A. Ostrowski, Über Quasianalytische Funktionen und Bestimmtheit Asymptotischer Entwickelungen, Acta Mathematica, vol. 53 (1930), p. 181.

The Rice Institute,

Houston, Texas 